\section{Adventures on the Dark Side: An Introduction to Darkfield Microscopy}

\author{
James E. Hayden \\ Bio-Graphics, Blue Bell, PA, USA
}

\section{INTRODUCTION}

Microscopists are used to looking at the world in the glaring light of brightfield illumination. When a specimen is placed on the microscope stage and the lamp is switched on, the general expectation is that the object will be seen transilluminated by a uniform, bright background. Light is expected to pass directly through the sample, forming an image that derives its colors and densities from the stains used during laboratory preparation.

Brightfield illumination is primarily used to examine sectioned and stained specimens. This lighting is adequate for routine diagnostic applications and in cases where a sample has enough inherent contrast to differentiate its component parts, but sometimes a sample is not stained, has no color, and cannot create enough contrast to be seen against the bright background-akin to trying to see details in a piece of plastic wrap placed on a light box. A different type of illumination may be necessary to create visual differences in the specimen's internal structures that are not as obvious in the direct light of brightfield. Occasionally, intriguing and creative microscopic landscapes are also revealed with a different set of lighting pathways. In situations such as these, darkfield illumination can provide a useful lighting alternative.

\section{SPECIMEN PREPARATION}

One of the primary reasons for using darkfield illumination is to identify faint or unstained specimens. Small organisms found in pond water, diatoms, hair fibers, and crystals all make excellent subjects. It is especially good at showing up any particulate matter on a slide. Immunological staining techniques that include conjugate metals such as silver or gold can be visualized easily. Fresh, wet-mount specimens such as blood, sperm, or cultured cells also show up well. These types of specimens generally have no inherent color and, being unstained, will remain colorless. With proper darkfield illumination, they will generally appear as white, glowing objects against a black background. Density differences in the specimens create varying amounts of reflection and refraction of the light passing through them, providing a continuous tonal range.

The most important aspect of specimen preparation for darkfield is to have impeccably clean glass slides. The same light that illuminates the specimen will also illuminate every bit of dust, dirt, grease, and excess mounting medium left on the slide. A smeared fingerprint on the glass will disperse the light so much that a black background may not be seen, even if the optical conditions are perfectly set. A thorough cleaning with a lint-free cloth dampened with ethanol will usually take care of most problems. A can of pressurized air is also useful to have on hand. After the slide is set on the stage, use the "soda straw" attached to the spray nozzle to blow away dust that has fallen after cleaning. A note of warning, though, for canned air: if the can is inverted or shaken, it will spray propellant, which will be deposited on the slide and require additional cleaning. It is also recommended to fire a blast of air before using it on the specimen to dislodge any particulate matter that might have been left in the nozzle.

\section{THE BASIC MICROSCOPE}

The conditions required to create darkfield illumination are fairly simple to produce, even if your microscope is not equipped with the necessary parts. To understand how to modify an existing instrument, it is necessary to understand first how the component parts of the microscope work and what is required to create the proper lighting pathways for brightfield illumination.

Figure 1 shows the relevant parts of the microscope to consider. The field diaphragm, substage condenser, condenser aperture, and objective lens are all important elements in creating both proper brightfield and darkfield illumination. The field diaphragm is a variable aperture that is located at the bottom of the microscope, generally at the point where the light comes out of the base. Its primary function is to control the amount of light reaching the condenser lens assembly. This is important because excess light passing through the condenser can reduce the quality of the image. As an analogy, imagine placing a $35-\mathrm{mm}$ slide in the middle of a large light box. All the extra light from the box creates a glare that makes it difficult to see the details in the transparency. If black card-

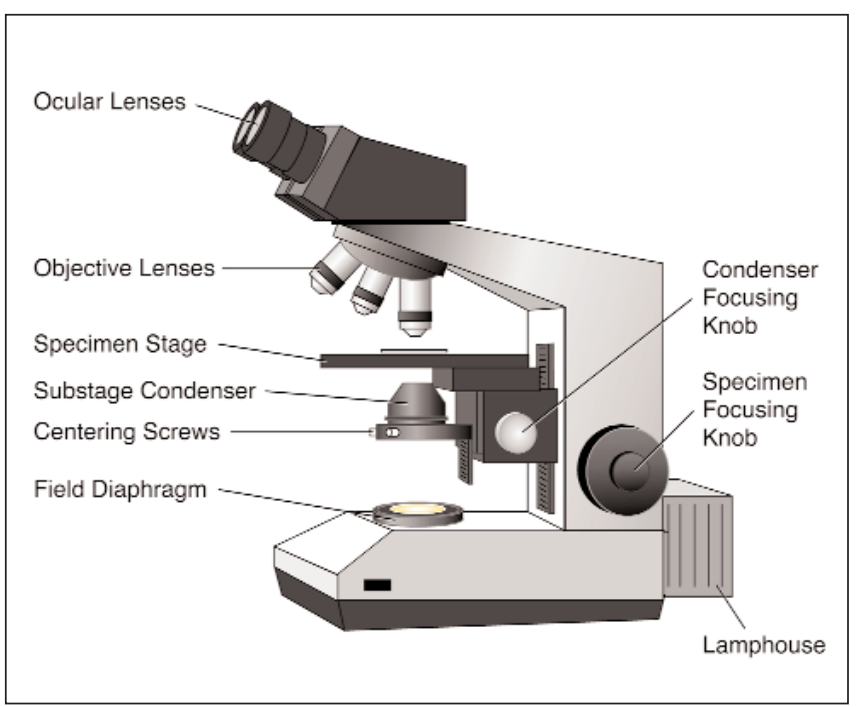

Figure 1. Generalized diagram of a compound microscope. 
board is placed all around the slide, then the excess light is blocked, making it easier to see the slide. The field diaphragm does the same thing on the microscope. When the proper lighting conditions are met, the field diaphragm blocks all light except just the amount needed to form the image.

Above the field diaphragm is the substage condenser. The condenser is mounted on a vertical focusing rail directly underneath the specimen stage and comes in a variety of forms. Some are composed of a sealed lens housing with a built-in aperture, some have a separate flip-up component, and some are built with a revolving turret that includes a variety of additional filter options. On some microscopes, there are also one or two swing-out filter carriers mounted just underneath the condenser lens. The primary function of the condenser lens is to collect and focus the illuminating beam at the proper plane in relation to the specimen.

The condenser aperture, sometimes called the condenser diaphragm or aperture diaphragm, is located inside the condenser housing and has two functions. First, like the field diaphragm, when closed down it restricts the amount of light passing through the condenser lens, eliminating excess light. In addition, as the aperture is further closed, it begins to refract the light passing through the condenser and can help to create visual shadows along the hard edges of the specimen. In this way, the diaphragm performs like a pair of squinting eyes, helping to visually sharpen the image and better see the sample. Care is required, though, as too much refraction can cause unwanted visual artifacts and actually reduce the detail seen. In keeping with the squinting analogy, there comes a point where, if you squint too hard, you cannot see anything.

Directly above the condenser is the specimen stage, which is nothing more than a movable platform with a hole in the middle. The specimen is placed over the hole so the light can pass through to the objective lens. The objective lens is then the primary magnifying lens in the system. It enlarges the image of the specimen as it continues on its way to the oculars, where the microscopist looks into the instrument, and/or the camera back where the image can be recorded. Objective lenses come in a variety of types, but the important features for this discussion are the magnification, written on the lens as $10 \times$, for example, and the numerical aperture (N.A.) of the lens, also inscribed on the barrel such as N.A. 0.25. For simplicity, it is only important to realize that each objective lets in a different amount of light and will require a different darkfield stop to create the darkfield illumination.

\section{KÖHLER ILLUMINATION}

Light in a microscope bulb is created with a glowing spiral of tungsten. Because of this uneven light source, the optical system of the microscope must be aligned in just the right way to provide the brightest, most even illumination possible across the specimen. This condition is met by setting Köhler illumination. By definition, Köhler illumination consists of focusing and centering the filament, focusing and centering the image of the field diaphragm, and then adjusting the condenser aperture to its optimal setting. This sounds rather complicated, but for purposes of this discussion, the filament can 
Table 1. Comparison of Required Diameters for Darkfield Stops and Sizes of U.S. Coins

\begin{tabular}{|c|c|c|}
\hline $\begin{array}{c}\text { Lens } \\
\text { Magnification }\end{array}$ & N.A. & $\begin{array}{c}\text { Stop Diameter } \\
(\mathrm{mm})\end{array}$ \\
\hline $1 \times$ & 0.03 & $25-30$ \\
\hline $2 x$ & 0.05 & $8-11$ \\
\hline $4 \times$ & 0.10 & $8-14$ \\
\hline $10 x$ & 0.40 & $16-18$ \\
\hline $20 x$ & 0.40 & $22-24$ \\
\hline \multicolumn{2}{|c|}{ Dime } & 18 \\
\hline \multicolumn{2}{|c|}{ Penny } & 19 \\
\hline \multicolumn{2}{|c|}{ Nickel } & 21 \\
\hline \multicolumn{2}{|c|}{ Quarter } & 24 \\
\hline
\end{tabular}

be left alone and the rest of the procedure only takes a few seconds to accomplish. Properly setting Köhler illumination is a prerequisite for correct brightfield and darkfield imaging.

Each objective has a different numerical aperture and lets through a different amount of light, so Köhler illumination must be reset each time a new objective is used. The procedure takes little time and, once mastered, is equivalent to tying your shoe, but explaining it in writing can be rather tedious. Start by placing a specimen on the stage and focusing it with the specimen focusing knob. Next, close the field diaphragm all the way down and focus the substage condenser with the condenser focusing knob (NOT the specimen focusing knob!) until you observe a distinct spot of light with sharp edges. This spot is the image of the field diaphragm, and it must be focused in the same plane as the specimen. At this point, recheck to see that both the spot and the specimen are in focus. If the edges of the diaphragm do not seem quite sharp, then it may be that the condenser is not focused far enough. Often, there is a color fringe as well. If one side of the spot has a blue edge and one side has a red edge, then the diaphragm is as focused as it will get.

After focusing the image of the field diaphragm, it must be centered. This is done with the two small centering screws (if present) on the front of the condenser support. If there are no screws, then loosen the condenser on its support, carefully nudge it until the spot is centered, and then tighten it down. While looking through the oculars, slowly open the field diaphragm until the edges of the spot just disappear from view, making small centering adjustments as necessary. If the microscope is properly aligned, then the condenser will not need to be centered again, but the field diaphragm will still need to be resized for each objective.

The final step in Köhler illumination is normally to optimize the opening of the condenser aperture. For darkfield illumination, this step can be eliminated because the condenser aperture must be opened to its maximum setting to let in as much light as possible. However, understanding the adjustment is useful, as the darkfield stop that is used to create the darkfield conditions needs to be placed in the same plane as the condenser aperture. To visualize this plane, remove an ocular from the microscope and look down the tube at the back objective focal plane. The condenser aperture can be seen at this point. Prove this by closing down the condenser aperture while looking in the tube, and the blades of the diaphragm can be seen moving.

\section{CREATING DARKFIELD}

Much effort has now been spent on describing conditions for brightfield illumination. This is necessary because the microscope optics must be set in specific planes to meet the requirements of darkfield, and this is also accomplished when setting Köhler illumination for brightfield. From a practical standpoint, first setting Köhler illumination provides a standard position that can be referenced and returned to while tweaking the optics to optimal settings. Figure 2 provides a simplified, side-by-side comparison of the light paths and results in both brightfield and darkfield.

Darkfield conditions are created when brightfield light originally destined to pass directly through the specimen and into the objective lens is blocked by an opaque darkfield stop on its way to or through the condenser. This stop must be of sufficient diameter to block the direct light passing through the condenser, but it must also be open around the edges, letting light pass by the outside of the stop. With the direct light blocked from entering the objective, the background field of view will then be black instead of white.

In Figure 2, note that the diameter of the darkfield stop is the same as the diameter of the aperture opening in brightfield. In the brightfield configuration, the cone of light that is created includes only the amount that would actually enter the objective lens. Any light that would be outside of this cone would be bent too far to enter the objective. The darkfield configuration takes advantage of this fact by blocking the center cone of

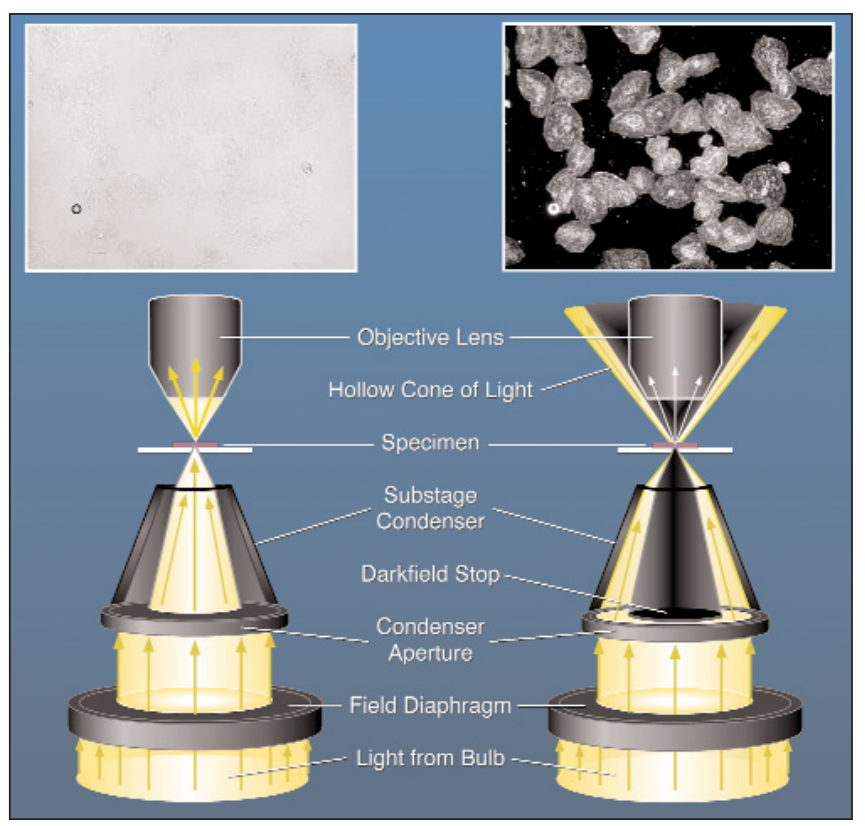

Figure 2. Schematic diagram illustrating a simplified comparison of the illumination pathways in brightfield (left) and darkfield (right) techniques. The micrographs are both of an unstained wet mount of buccal epithelial (cheek) cells. Both images are of the same field. 
light with the darkfield stop and opening the condenser aperture to allow the outside light to pass. The light that is allowed to pass by the darkfield stop is refracted at an oblique angle inside the condenser and would normally pass just outside the objective lens in a hollow, inverted cone of light.

If a specimen is in place on the stage, then it would be located at the apex of the hollow cone of light. All of the light passing through would strike the specimen. This light would then be scattered in all directions, but some would be reflected or refracted upward into the objective lens, resulting in a bright image of the specimen seen against the black background.

\section{MATERIALS AND METHODS}

The equipment requirements for proper darkfield illumination are minimal and depend on what is already available. If one is fortunate enough to have a phase turret condenser installed on the microscope with a darkfield stop included, then darkfield studies can begin at once. The turret usually has five or six openings, with one being reserved for brightfield (this setting includes a sliding ring to adjust the condenser aperture), one opening has a darkfield stop (marked as DF), and then there are several phase stops (marked as PH1, PH2, or $\mathrm{PH} 3$ ). The turret condenser is the best condenser option because the darkfield stop is placed in exactly the same location as the condenser aperture, where it is supposed to be according to the light path diagrams. If the turret does not include a darkfield stop, or one of a different size is needed, one can be made to fit into any of the free openings.

If a turret is not available, then any condenser on a focusing rack will do. However, darkfield stops will have to be devised. All that is needed is some opaque material that can be easily cut into small circles, like a heavy foil, and something to support it that lets light get past, like a glass microscope slide or clear acetate. Ideally, the darkfield stop needs to be located in the plane of the condenser aperture, but if the aperture is enclosed inside a sealed housing, then the stop should be placed as close as possible underneath the condenser. If the condenser includes a swing-out substage filter carrier, then the stop can be placed in that; otherwise, it can be taped to the bottom of the condenser itself (note: do NOT tape to any of the glass optics inside the lens, just to the external housing).

Low-magnification microscopy generally requires special low-magnification condensers to achieve optimal lighting conditions. Lenses in the $1-2 \times$ range that are less than 0.10 
N.A. fall into this category. Fortunately, from a practical point of view for those who do not have such lenses, very good results can still be obtained by removing the condenser lens altogether. In this configuration, the field diaphragm behaves as the condenser aperture, and darkfield illumination can be easily achieved by resting the correct stop directly over the field diaphragm. This technique will not work for lenses with higher numerical apertures because the angle of the light entering the objective will not match the necessary requirements to produce the hollow cone of light.

Before anything can be attached, however, the stop needs to be fabricated. As previously mentioned, each lens will have its own size requirements based on the numerical aperture, but there is some overlap. Size will also be affected by the exact location of the stop. For the purposes of this article, objective lenses in the low-to-medium magnification range $(2-10 \times$ or $0.05-0.25$ N.A.) work best. The rules can also be applied to $1 \times$ or $20 \times$ lenses, but it gets trickier to get the precise alignments. High magnifications (40X and above or anything above 0.65 N.A.) require special darkfield condensers that are available from manufacturers for their specific microscopes. An excellent source for additional information about these condensers and darkfield illumination in general can be found at http:// www.microscopy.fsu.edu/primer/techniques/darkfield.html.
To make the darkfield stops, first rotate a lens into position (start with the $10 \times$ lens), place a specimen on the stage, and set Köhler illumination. Next, place a clear millimeter ruler in the location where the stop will be. Remove an ocular and look down the barrel toward the back objective focal plane; the ruler should be visible. Center the ruler so that the diameter of the light in the tube can be read directly. The darkfield stop will need to have this diameter as a minimum.

Cutting the stop requires an accurate circle at the start. Look around for any small, circular object that can be used to trace the circle, and use it as a template. Then, carefully cut the stop out. If the diameter is close, then you may even be able to use a coin as the stop. Table 1 provides a starting point for approximate darkfield stop sizes.

Once the darkfield stop is cut, affix it to a transparent support with glue or clear tape. A microscope slide or piece of clear acetate, such as a developed piece of unexposed sheet film, works well for this. Place the whole arrangement in the predetermined location on the microscope. Center the stop by
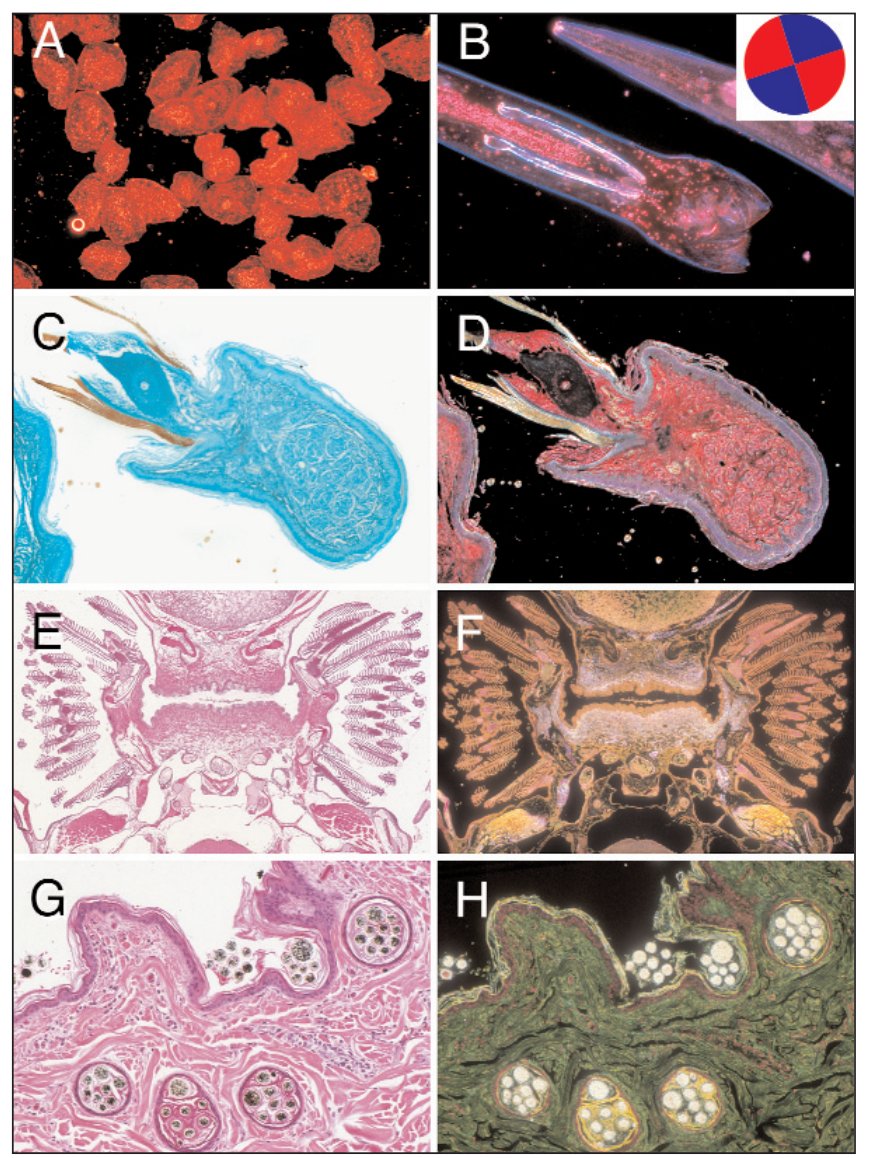

Figure 3. Examples of darkfield illumination utilizing color. (A) Buccal epithelium with darkfield illumination as shown in Figure 2 with the addition of a red filter to the light path. (B) Male $O$. ostertagi shown in darkfield with the addition of the filter shown in the inset. (C and D) Mouse toe saggital section stained with Gomori methenamine silver (GMS) stain and shown in both brightfield and darkfield. (E and F) Cross section of zebrafish head through the gills stained with hematoxylin and eosin (H\&E) and shown in both brightfield and darkfield. ( $\mathrm{G}$ and $\mathrm{H}$ ) Section of canine epidermis with cross sections of compound hair follicles stained with H\&E and shown in both brightfield and darkfield. 
looking down the barrel of the microscope tube with the ocular removed and fix it into place. Replace the ocular and, if all works correctly, darkfield lighting will have been achieved. Unfortunately, because of the precise requirements, it will probably be off a bit. Depending on how close the stop is to the condenser aperture, you may need to adjust the condenser focus up a bit, need a different size diameter stop, or need to fine-tune the centering. All of this can best be done while observing the effects through the oculars.

\section{COLOR IN DARKFIELD}

Specimens best observed with darkfield techniques are usually colorless and form white-on-black images with very little obvious color. This monochromatic thinking does not mean that there is no place for color in darkfield. On the contrary, there are many situations where the use of color, from original stained specimens or introduced via filters in the light paths, can produce useful and stunning images.

Adding color by inserting filters into the light path is very straightforward. Simply place any colored, translucent object directly over the field diaphragm, and that colored light would then illuminate the specimen. The filter does not even need to be a "real" filter; any colored, translucent item will do. Specialized filters can even be made by coloring on clear acetate with colored markers. An unstained specimen would then take on the color of that filter. Figure 3A shows the results of placing a red filter in the light path while photographing the buccal epithelium shown in Figure 2. Notice that, while it definitely has color, it is still essentially monochromatic.

Creative use of filters can greatly enhance the information seen in an image. By using different colors at the same time and arranging them in alternate patterns, the directionality of the specimen's internal structures can be made to stand out. In Figure $3 \mathrm{~B}$, the male round worm Ostertagia ostertagi was prepared as a wet mount with an unstained, fixed sample. The filter used was a circle divided into quarters, with alternate quarters being blue and red. With darkfield lighting established, this filter was placed in the light path with the orientation shown in the inset, allowing the blue light to approach the specimen from two directions $180^{\circ}$ apart. The parts of the worm that came close to the same directional plane as the blue part of the filter then reflected the blue light strongly into the objective, illuminating those parts of the specimen blue. The red was the brighter part of the filter and thus tended to illuminate everything else that was not already strongly blue. Where the amount of scattered light is most intense, the color is washed out and the structure appears white. By rotating the filter in relation to the specimen, a highly differentiated result was obtained.

A specimen that already has been stained or has other inherent color can also show a variety of colors in darkfield, but they are generally not the same colors seen under brightfield conditions. Figure 3, C-H, are comparisons of brightfield and darkfield illumination of specimens that were first stained histologically. In each example, notice how the original colors have been altered by the darkfield technique. These color shifts are caused by a variety of independent factors and are often difficult to predict in advance. In each case, however, the original white light falling on the specimen was refracted through the colored tissue, and the wavelength shifted as a consequence. The perceived color of the light was affected by such factors as the original hue of the stain, the thickness of the specimen, and the density of the tissue. Harder, denser tissues tend to reflect light more than refract it, so those parts of the tissue tend to be darker and closer in wavelength to the original color. Softer tissues refract the light much more and alter the wavelengths in greater amounts, resulting in a stunning variety of colors. In this way, the colors produced not only provide an aesthetically pleasing result but also give some clues as to the internal characteristics of the tissues.

\section{CONCLUSIONS}

Darkfield illumination is a simple, inexpensive contrast enhancement technique for light microscopy. It allows faintly visible specimens to be seen, greater differentiation of internal structures, and stunning color renditions that are not only beautiful but also convey additional information. The equipment required is minimal, easy to create, and simple to modify. When specimens are obtained that require special attention, a glance in darkfield conditions may provide useful and unexpected results.

\section{FURTHER READING}

1.Abramowitz, M. 1987. Contrast Methods in Microscopy: Transmitted Light. Olympus Corp., Lake Success, NY.

2.Delly, J.G. 1988. Photography through the Microscope. Eastman Kodak Company, Rochester, NY.

3.Lawson, D. 1972. Photomicrography. Academic Press, New York.

4.Vetter, J.P. 1992. Photomicrography, p. 137-170. In J.P. Vetter (Ed.), Biomedical Photography. Butterworth-Heinemann, Stoneham, MA.

Address correspondence to James E. Hayden, BioGraphics, 1058 Hemlock Drive, Blue Bell, PA 19422,USA.e-mail: jhaydenrbp@earthlink.net

\section{Suggestions for contributions to the Biolmaging feature are welcomed by its editor, Dr. Steve Paddock (paddock@facstaff.wisc.edu)}

